\title{
COVID-19 Pandemic: Increased risk for psychopathology in Children and Adolescents?
}

\author{
Esther Via ( $\nabla$ evia@sjdhospitalbarcelona.org) \\ Hospital Sant Joan de Déu of Barcelona, Spain https://orcid.org/0000-0002-2331-3232 \\ Xavier Estrada-Prat ( $\square$ xestrada@sjdhospitalbarcelona.org ) \\ Hospital Sant Joan de Déu of Barcelona, Spain \\ Jordina Tor \\ Institut de Recerca Sant Joan de Déu, Barcelona, Spain \\ Carles Virgili \\ Independent researcher in col. Hospital Sant Joan de Déu of Barcelona, Spain \\ Marina Fàbrega \\ Hospital Sant Joan de Déu of Barcelona, Spain

\section{Laura Duran} \\ Psychiatry and Legal Medicine Department. Universitat Autònoma de Barcelona. Barcelona, Spain. \\ José A Alda \\ Hospital Sant Joan de Déu of Barcelona, Spain \\ Charlotte Keating \\ Swinburne University of Technology. Hawthorn, Australia \\ Montserrat Dolz \\ Hospital Sant Joan de Déu of Barcelona, Spain
}

\section{Research Article}

Keywords: COVID-19, stress, psychopathology, children, adolescents

Posted Date: November 11th, 2020

DOI: https://doi.org/10.21203/rs.3.rs-104507/v1

License: 두 (i) This work is licensed under a Creative Commons Attribution 4.0 International License. Read Full License 


\section{Abstract}

COVID-19 pandemic is prompting multiple stressors -including control strategies such as lockdown- which may impact child and adolescent mental health. 1,529 caregivers answered an online questionnaire about emotional and behavioral symptoms of youths (4-18 years old) using the Pediatric Symptom Checklist (PSC). Percentage of above-the-risk-threshold PSC scores (PSC+) were compared with a baseline measure. Associations between lockdown PSC scores and selected variables were evaluated using a linear regression analysis. PSC+ significatively increased from $13 \%$ to $34.7 \%$, baseline to lockdown, mostly driven by depression and anxiety symptoms and with greater risk at younger ages. Youths' and parents' positive mental health history additionally increased this risk. In children, caregivers' stress and depression was the stronger predictor of lockdown PSC scores; in adolescents it was the coping style. These findings suggest a significant mental health impact on children and adolescents associated with COVID-19 pandemic response. Younger ages should be closely monitored.

\section{Introduction}

The emergence of the COVID-19 disease, produced by the new SARS-CoV2 coronavirus first described in Wuhan (China) in December 2019, has been declared a pandemic since 11th March [1]. Five months later, it has generated more than 21 million infections world-wide and is having significant impact, medically, socially, and economically. From east to west, every country has applied preventive strategies to minimize the number of cases, such as social distancing policies [2]. Spain is one of the most affected European countries, with more than 400.000 infections and almost 29.000 deaths [3]. While Spain established a stringent and wide-spread lockdown -lasting up to six weeks- to control the spread of the virus (see [2] and Figure1), many have raised concern regarding the putative consequences for mental health, particularly in vulnerable groups such as children and adolescents $[1,4]$. Early reports have revealed huge distress, anxiety and depression in some of these groups [5], but little is known about the psychological impact specifically for youths [6-8].

The COVID-19 crisis is characterized by different and coexisting multifaceted stressors. In children and adolescents, they include the risk of youth's own or relatives' infection or even a relative's death, but more likely the fear of contracting the virus. Indeed, the uncertain COVID-19 scenario is likely worsened by the dissemination of ambiguous information, which is known to increase emotional distress [9]. In addition, income reduction and job destruction have increased the risk of social status loss, which might impact youths, through increased parental worries and anxiety, including concern about covering basic needs [10]. More importantly, school closures have affected more than one thousand million students worldwide, forcing learning from home despite the digital gap [11]. This measure involves a change in routines and care, a lack of contact with peers, and even in some cases a lack of ensuring a meal per day or even a safe environment [12]. For those youths with a mental health diagnosis (5\% below 18 years old in our environment [13] another significant stressor is the reduction in accessible mental health resources [14].

When faced with stressful events, normal, age-dependent responses of children and adolescents involves becoming anxious and fearful, but also irritable, numb, restless, the possibility of developing headaches, stomachaches or neurofunctional alterations; in some cases these symptoms might develop into a mental health disorder [15]. Hypotheses for mental illnesses are based on a complex interaction between genes and environment [16]. For example, stressors, particularly those occurring at early life stages, are considered to involve negative consequences on neurodevelopment and increase vulnerability for a mental illness in adulthood [17, 18]. A prevalence of around $15-30 \%$ in anxiety and depressive disorders have been reported in adult and adolescent studies assessing the incidence of mental illness in natural and epidemic catastrophes [19-21]. When younger samples have been included, a more severe stress response has been reported, with symptoms being even four times higher in children [20,22]. Additionally, in the context of COVID-19, social isolation might worsen anxiety, depression and stress [4]. The first COVID-19 studies in China found increased anxiety, stress, and depression symptoms in adolescent females, with a $39.5 \%$ of this population meeting criteria for clinical depression [7, 8]. In another sample of children and adolescents, depression symptoms were significant on $22 \%$ of respondents, with greater risk associated with female sex, emotion-focused coping style and being an urban resident [23]. One European study evaluated 1,143 parents of youths on the effects of lockdown, finding an increase in anxiety- and stress-related symptoms, -but did not evaluate risk for psychopathology based on a validated scale- [24]. 
In the current COVID-19 health emergency it is mandatory to identify vulnerable groups to anticipate mental health strategies. Childhood and adolescence confer greater vulnerability, that might be worsened by low socioeconomic status, the presence of negative life events or a psychiatric diagnosis [4,25]; specific interventions based on those identified modifiable factors might help youths to cope with stress [26, 27]. Studies assessing mental health in confinement periods are scarce and mostly in adult non-European samples (see [28]). This study aims to evaluate emotional and behavioral symptoms in 4-18 years old subjects to identify those at a higher risk for a mental disorder through the use of a validated screening questionnaire. Another study aim is to identify potential risk and protective factors for psychopathological symptoms. We expect around 15 to $30 \%$ of participants to demonstrate increased risk for a mental illness with a predominance of anxiety and depressive symptoms. We additionally hypothesize increased risk associated with younger age, being female and of low socioeconomic status.

\section{Methods}

\section{Participants}

We conducted an online survey addressed to parents or legal guardians (from now on, caregivers) of children and adolescents between 4-18 years (EmCoVID19 study). The survey was aimed at Spanish participants; the only requirement was for caregivers to understand this language. A predominantly Catalan sample was expected, as per recruitment strategies (see further). A minimum of 1200 participants were required, considering the most similar study at the time of study design [7].

\section{Survey Development}

The survey was built on Research Electronic Data Capture system, REDCap [29]. An http link leading to the survey was automatically generated and distributed through active and passive recruitment strategies. Active strategies were based on probabilistic sampling, by contacting all Catalan schools (government and privately funded) and asking them to send survey invitations to caregivers. Passive strategies involved non-probabilistic approaches: snowball sampling (i.e. contacting caregivers through social media -Whatsapp ${ }^{\mathrm{TM}}$ - and asking them to contact others) and institutional advertisements (i.e. the Hospital Sant Joan de Déu's website or Twitter ${ }^{\mathrm{TM}}$ ). To encourage participation, recommendations for dealing with stress based on the obtained score responses were sent to the caregivers' personal email obtained via the survey. The survey was open for recruitment from May 6th to July 6th 2020.

\section{Measures}

Main outcome variable: PSC

Emotional and behavioral symptoms were measured with the Pediatric Symptom Checklist (PSC) Spanish version, a 35-items screening questionnaire informed by caregivers about four different dimensions: depression, anxiety, hyperactivity and conduct (see TextS1)[30]. The PSC checklist was administered twice. First, to evaluate symptoms during the last year but prior to population lockdown (baseline PSC). Second, to evaluate symptoms since the start of lockdown to the time of survey response (lockdown PSC).

\section{Secondary variables}


We collected sociodemographic variables such as sex, age and socioeconomic status -5 levels defined by the Hollingshead scale [31]Other variables included COVID-19 related questions (outbreak severity perception, being in lockdown at the time of the survey, a COVID19 diagnosis in the home environment, leaving home for work, establishment and accomplishment of routines), changes in biorhythms, mental health diagnosis history in children/adolescents or in caregivers (Table1, TableS1). To evaluate stress and depressive symptoms in caregivers, we included six items of the Depression, Anxiety and Stress Scale (DASS-21, [32](TableS1 for items reference). We evaluated stressful life events (SLE) that occurred to the child/adolescent at two different timeframes, akin to PSC: baseline SLE and lockdown SLE. They were evaluated using the Social Readjustment Scale for children, a 43-item that produces a weighted-for-stressorseverity score (weighted by life change units (LCU)) [33,34] (see TextS1). Finally, youths' coping strategies were assessed using the Coping Scale for Children (Escala de afrontamiento para niños, [35]), a 36-item scale that was adapted to be completed by caregivers. The scale includes questions about the pattern of response in four different contexts, and for the purpose of this study, we included two of these contexts (problems at home, problems with peers at school). The original scale is validated for children/adolescents between 912 years old and we only used the responses for the adolescent group (>10 years old, see Table1 and TableS1 for all variables).

\section{Ethical considerations}

The study was approved by the Biomedical and Ethics Research Committee of the Hospital Sant Joan de Déu in Barcelona and complies with the principles outlined in the Helsinki Declaration (64th World Medical Association General Assembly, Fortaleza, Brazil, October 2013). Caregivers were asked to sign the informed consent prior to initiating the survey. When the adolescent was 12 years old or older, caregivers were asked to obtain consent from the adolescent.

\section{Database preprocessing and statistical analyses}

Data stored in REDCap was downloaded, incomplete or duplicated data were eliminated. Descriptive and statistical analyses were conducted using IBM SPSS Statistics for Windows v.25.0 (IBM Corp. Armonk, NY: IBM Corp.). An alpha risk of 0.05 was assumed. When variables were exploratory, an alpha risk of 0.001 was assumed.

Descriptive statistics were used to provide sociodemographic and COVID-19-related sample characteristics, percentage of baseline PSC+ and lockdown PSC+, central tendency statistics for the SLE scale and the coping scale. DASS-21 subscores were described with central tendency statistics and percentages for each item.

Pediatric Symptom Checklist (PSC): all participants and groups of interest

A McNemar analysis was used to evaluate changes in PSC+ percentages from baseline to lockdown.

We evaluated those variables that differentiated children/adolescents with a PSC+ from the rest of the sample (PSC-). Selected variables included: sex (male/female), age (children/adolescents -cut-off at 10 years old, [36]), socioeconomic status (5 levels), the presence of a positive psychiatric history in the child/adolescent, the presence of a positive psychiatric history in caregivers, DASS-21 scores, lockdown LCU and coping style scores. Other exploratory variables included are described in TableS2. Chi Squared or one-way analysis of variance (ANOVA) tests were used when required. Predominance of symptom's dimensions were described and compared by sex and age groups.

\section{Predictors of lockdown PSC scores}

We conducted two different linear regression analyses dependent on the age (children's group, 4-9 years old, adolescents' group 10-18 years old); this decision was driven by the coping scale, which could not be evaluated in children. The independent values were the DASS-21 subscores, positive history of psychiatric diagnosis in youths, the weighted SLE score (LCU), and the coping scale only for the adolescent's regression.

\section{Results}




\section{Participants}

There were 5,929 entries to the survey. Of these, 1,666 provided informed consent and an email contact in a first step and were thus included in the study. From the 1,666, we excluded participants not fulfilling inclusion criteria (i.e. age range, $n=86$ ) and duplicates $(n=51)$. The final sample included 1,529 participants. Participants were mostly from Catalonia (93.0\%), with a mean age of 8.49 years old (SD 3.42; range 4-18 years old). Most of the sample were children (66.58\%). Sex was evenly balanced in the sample (males:53.9\%). The sample included mostly families with upper-middle and upper socioeconomic status (82\%) (Table1).

\section{Descriptive results}

The majority of respondent families were in lockdown at the time of completing the survey $(71.7 \%)$. More than half of the caregivers left home for work $(n=561 ; 54.4 \%)$. Most of the children and adolescents performed online classes during school closures $(n=958 ; 66.8 \%)$, while others were not receiving either online or in-person classes $(n=458 ; 31.9 \%)$; instead, a small group were attending school $(n=19$; $1.3 \%$ ). Sixty-four families (4.4\%) reported that one or more family members presented COVID-19 symptoms or a formal COVID-19 diagnosis. A high percentage of caregivers reported being concerned at a moderate or high level about COVID-19 crisis ( $n=1312 ; 85.8 \%)$; in children/adolescents, this percentage -as reported by caregivers- was found in only $59.2 \%$ participants (children: $n=274 ; 28.4 \%$; adolescents: $n=214 ; 44.7 \%)$. TableS1 shows other relevant variables of the entire sample.

Stress and depression in caregivers and Stressful life events (SLE)

DASS-21 scale results and SLE are presented in Table1 and TableS1. For DASS-21, the item with the larger proportion of significant ratings was "finding it hard to wind down" (i.e. $42.9 \%$ of caregivers rated it as "often" or "almost always"). For SLE, $45.5 \%$ participants reported experiencing no SLE, others reported between one to three SLE (50.5\%), and $4.1 \%$ reported four or more SLE. The weighted measure -LCU- showed a mean score of 62.92 (SD=94.37) for baseline SLE and 43.58 (SD=51.66) for lockdown SLE.

\section{Coping style of adolescents}

Most of the participants presented a coping style focused on the problem (53.6\%), while a $38.8 \%$ reported a tendency toward an unproductive coping style. A minor percentage (7.5\%) reported unproductive or productive coping strategy depending on the situation (Table1).

\section{Table 1. Sample clinical and sociodemographic characteristics-l.}




\begin{tabular}{|c|c|c|c|}
\hline & $\mathrm{N}$ & $\mathrm{n}(\%)$ & mean(SD) \\
\hline Age & 1,529 & & $8.49(3.42)$ \\
\hline Children(4-9 years old) & & $1,018(66.58 \%)$ & $6.48(1.70)$ \\
\hline Adolescents(10-18 years old) & & $511(33.42 \%)$ & $12.49(2.30)$ \\
\hline Sex & 1,529 & & \\
\hline Female & & $705(46.11 \%)$ & \\
\hline Male & & $824(53.89 \%)$ & \\
\hline Family Style & 1,481 & & \\
\hline Single-parent & & $129(8.71 \%)$ & \\
\hline No single-parent & & $1,352(91.29 \%)$ & \\
\hline Socio-economic status(SES) & 1,401 & & \\
\hline 8-19(lower) & & $27(1.99 \%)$ & \\
\hline 20-29(lower-middle) & & $70(5.00 \%)$ & \\
\hline 30-39(middle) & & $155(11.06 \%)$ & \\
\hline 40-54(upper-middle) & & $453(32.33 \%)$ & \\
\hline 56-66(upper) & & $696(49.68 \%)$ & \\
\hline \multicolumn{4}{|l|}{ History of mental health problems } \\
\hline Children/adolescents(yes/no) & 1,282 & $88(6.86 \%) / 1,194(93.14)$ & \\
\hline Parents(one or both/no/unknown) & 1,279 & $97(7.39 \%) / 1,182(90.09 \%) / 33(2.51 \%)$ & \\
\hline DASS-21 subitems & 1,401 & & \\
\hline Total DASS-21 subitems score & & & $6.61(3.37)$ \\
\hline Coping style(10-18 years old) & 520 & & \\
\hline Focused on the problem & & $214(53.63 \%)$ & 19.36(3.80) \\
\hline Unproductive & & $155(38.85 \%)$ & 18.26(3.68) \\
\hline No predominance & & $30(7.52 \%)$ & \\
\hline Stressful life events(SLE) & 1527 & & \\
\hline LCU since lockdown & & & $43.58(51.66)$ \\
\hline Ratio LCU since lockdown/month & & & $19.88(23.85)$ \\
\hline LCU last year & & & 62.92(94.37) \\
\hline Ratio LCU last year/month & & & $5.24(7.87)$ \\
\hline
\end{tabular}

\section{Main outcomes}

Only for these analyses, two additional non-Spanish participants were excluded due to unknown information regarding whether they had been in lockdown ever.

Pediatric Symptom Checklist (PSC): all participants

Thirteen percent of participants received a clinically significant score for the baseline PSC (PSC+). This figure increased to $34.7 \%$ during lockdown. Baseline to lockdown PSC scores' differences were statistically significant (McNemar Chi Squared=218.89; $p<.001 ; O R=11.2$; IC 95\%(7.46-17.37)). Additionally, 23.8\% changed from a baseline PSC- to a lockdown PSC+ and 2.1\% improved, changing from a 
baseline PSC+ to a lockdown PSC-. Lockdown PSC+ scores were mostly driven by depression and anxiety symptoms (64.5\%), more than by hyperactivity and conduct symptoms (30.8\%) or mixed symptoms (4.7\%) (TableS3).

Pediatric Symptom Checklist (PSC): groups of interest

Regarding age, the percentage of lockdown PSC+ was significantly higher in children (39.4\%) compared to adolescents (25.2\%) $\left(X^{2}(1)=23.88 ; p<.001 ; O R=1.93 ;\right.$ IC 95\%(1.48-2.51)). For those with lockdown PSC+, depression and anxiety were more frequent in adolescents than children $\left(X^{2}(1)=7.15 ; p=.007 ; O R=1.97\right.$, IC 95\%(1.19-3.27)) whereas hyperactivity and conduct were more frequent in children compared to adolescents $(X 2(1)=6.59 ; p=.010 ; O R=1.99$, IC 95\%(1.17-3.39)), but differences were not statistically significant $(p<.001)$. There were no age-related PSC+ differences at baseline $\left(X^{2}(1)=.58 ; p>.05\right)$; despite SLE were associated with PSC scores (see further), when no SLE occurred, the higher risk for children remained significant $(X 2(1)=11.93 ; p=.001 ; O R=2.40 ;$ IC 95\%(1.45-3.98)) (Table2 and TableS5 for age-related sociodemographic characteristics).

In a post-hoc analysis, a Receiver operating characteristic (ROC) analysis was conducted to evaluate the age cut-off for greater risk for a lockdown PSC+. The 8.5-years-old cut-off model showed the best predictive power, despite low validity ( $p<.001, \mathrm{AUC}=.58, \mathrm{IC} 95 \%$ (.55-.61), Sensitivity 67\%, and Specificity 48\%) (FigureS1).

There were no sex-related differences in the percentages of lockdown PSC+ (Males: 36.9\%, Females: 32.1\%; X2(1)=3.11; $p>.05$ ). Depression and anxiety symptoms were more frequent in females than males $(X 2(1)=18.58 ; p<.001 ; 0 R=2.53$, IC 95\%(1.65-3.89)) and hyperactivity and conduct symptoms were more frequent in males than females $(X 2(1)=14.02 ; P<.001 ; 0 R=2.32$, IC 95\%(1.48-3.61)). Percentages of PSC+ during the lockdown were not different across the five socioeconomic levels $(X 2(4)=3.24 ; p>.05)$.

A higher risk for a lockdown PSC+ was found for youths with a personal history of a mental illness $(X 2(1)=34.97 ; p<.001 ;$ OR=3.71, IC $95 \%(2.34-5.88)$ ) and when one or both caregivers had a mental illness history $(X 2(1)=13.31 ; p<.001 ; O R=2.22$, IC 95\%(1.43-3.43)). In both cases, these differences were already present at baseline (positive history in youths: $(X 2(1)=101.71 ; p<.001 ; 0 R=8.20, I C 95 \%(5.13-13.09)$; positive history in one/both caregivers: $(X 2(1)=28.23$; $p<.001$; OR=3.39, IC 95\%(2.11-5.45). The PSC+ group presented higher DASS-21 subscores, an unproductive copying style mean and a higher score for the stressor-related lockdown LCU than the PSC- group (Table2).

Table 2. Main clinical and sociodemographic comparisons between PSC+ and PSC-. 


\begin{tabular}{|c|c|c|c|c|c|c|}
\hline & & lockdown PSC- & lockdown PSC+ & $\mathbf{p}$ & $\begin{array}{l}\mathrm{Chi}^{2} / \mathrm{T}- \\
\text { student }\end{array}$ & $\begin{array}{l}\text { OR, IC } \\
95 \%\end{array}$ \\
\hline & $\mathrm{N}(\%)$ & $\mathrm{n}(\%)$ or mean (SD) & $\mathrm{n}(\%)$ or mean (SD) & & & \\
\hline Sex (female/male) & $1216(79.63 \%)$ & $375(67,93 \%) / 419(63,1 \%)$ & $177(32,07 \%) / 245(36,9 \%)$ & .08 & 3.11 & \\
\hline $\begin{array}{l}\text { Age } \\
\text { (children/adolescents) }\end{array}$ & $1216(79.63 \%)$ & $492(60,59 \%) / 302(74,75 \%)$ & $320(39,41 \%) / 102(25,25 \%)$ & $<.001$ & $23.88^{*}$ & $\begin{array}{l}1.93(1.48- \\
2.51)\end{array}$ \\
\hline \multicolumn{7}{|l|}{$\begin{array}{l}\text { History of a } \\
\text { psychiatric diagnosis }\end{array}$} \\
\hline in youth (yes/no) & $1190(77.93 \%)$ & $31(36.47) / 752(68.05)$ & $54(63.53) / 353(31.95)$ & $<.001$ & $34.99 *$ & $\begin{array}{l}3.71(2.34- \\
5.88)\end{array}$ \\
\hline in caregivers (yes/no) & $1189(77.87 \%)$ & $42(47.73) / 737(66.94)$ & $46(52.27) / 364(33.06)$ & $<.001$ & $13.31^{\star}$ & $\begin{array}{l}2.22(1.43- \\
3.43)\end{array}$ \\
\hline $\begin{array}{l}\text { DASS-21 Subscores } \\
\text { total }\end{array}$ & $1203(78.78 \%)$ & $6,0405(3,18)$ & $8,527(3,42)$ & $<.001$ & $-10.69 *$ & \\
\hline $\begin{array}{l}\text { Copying style: } \\
\text { problem centered } \\
\text { (positive values)- } \\
\text { unproductive } \\
\text { (negative values) }\end{array}$ & $1092(71.51 \%)$ & $2,4338(5,78)$ & $-3,8296(5,54)$ & $<.001$ & $-7.62^{\star}$ & \\
\hline $\begin{array}{l}\text { Stressors-lockdown } \\
\text { LCU }\end{array}$ & $1216(79.63 \%)$ & $45,7798(49,02)$ & $76,9713(59,01)$ & $<.001$ & $-4.76^{\star}$ & \\
\hline $\begin{array}{l}\text { Baseline PSC scores } \\
\text { (last year) }\end{array}$ & $1216(79.63 \%)$ & $12,25(6,97)$ & $27,16(8,11)$ & $<.001$ & $-43.42^{\star}$ & \\
\hline
\end{tabular}

Exploratory analyses showed that youths with PSC+ were reported to present more preoccupations about COVID-19, were less likely to have accomplished routines, and more likely to experience changes in appetite and sleep patterns than youths with PSC- (TableS2).

\section{Predictors of lockdown PSC scores}

Regarding the children's group, the linear regression analysis showed three different significant predictive PSC score models during lockdown. Both prior psychiatric diagnosis history in children and lockdown LCU scores positively predicted PSC scores; however, stress and depressive symptoms in caregivers was the strongest individual predictor in the model $(\beta=.39, p<.001)$. In the adolescents group, there were four different models including all the variables; coping style was the best predictor explaining PSC score variance ( $\beta=.44$, $\mathrm{p}<.001$ )(Tables4, Figures2).

\section{Discussion}

We found that $34.7 \%$ of children and adolescents between 4-18 years old might be at risk of developing a mental health problem in the context of lockdown due to the COVID-19 emergency. These percentage was mostly driven by depression and anxiety symptoms, but in one three, there was a predominance of hyperactivity and conduct symptoms. There was an age-effect in our sample, with an almost doubled increased risk in children compared to adolescents for mental health problems; with 8.5 years old representing the best threshold for the risk shift. In the children's group, caregivers' stress and depressive symptoms were the variables that better predicted child symptoms, followed by the presence of a mental illness in children and the number and severity of stressors since lockdown. In the adolescents' group, the best predictor was the coping style, although the rest of variables (caregivers' stress and depression symptoms, number and severity of stressors, presence of a psychiatric diagnosis history in adolescents) were also found to positively predict symptoms since lockdown. 
As anticipated, we found a significant increase in the percentage of children and adolescents at risk for a mental illness in lockdown. This represents a more than 2 -fold increase relative to what was retrospectively reported for the 12 months prior to the lockdown (13\%), in the context of expected levels in the general population samples (5-25\% [37]). The lockdown increase up to $34.7 \%$ is on the higher threshold of what we predicted, according to prior literature -mostly in adult and adolescent populations- in similar contexts (i.e. [21]). Despite a predominance of depressive and anxiety symptoms (65\%), there was a considerable $30 \%$ of predominance of hyperactivity and conduct. This was more prevalent in children, which might be partially explained as an anxious hyperarousal in younger children with limited emotional understanding and cognitive and/or language skills [38]. This finding is probably related to the large number of young children in our sample in contrast with other studies, where younger children are frequently underrepresented [23]. We did not find an increased rate of clinically significant scores in the females' group; however, anxiety and depressive symptoms were more prevalent in females, whereas hyperactivity and conduct alterations were more prevalent in males, a well stablished psychopathologic pattern [39]. These might explain the significant sex-effect in other studies, which used depression and anxiety-related scales as a measurement tool [7].

According to our results, children have been more affected than adolescents by the lockdown during the COVID-19 crisis. There are potentially different reasons for this. First, the current context has required an abrupt change in daily routines. Due to the immature stage of executive (e.g. planning, problem solving) and self-regulatory functions in children, they are in a greater need of external support [40]. Consistent with this, when routines were not accomplished in our sample, there was an increased risk for a significant score in the screening scale. Second, changes in socialization and learning patterns might have disproportionately impacted children. Developing and maintaining friendships is one of the most important milestones for children and adolescents (which can impact well into adulthood) [41]. The way young school children develop and maintain friendships is likely to be more interactive and play-based, and likely to involve engaging with friends on social media compared with their adolescent counterparts. In the same vein, online learning has been disproportionately challenging, as children are not developmentally ready, typically, to learn in an independent, self-directed manner. Third, the greater degree of emotional and physical independence of adolescents could confer greater protection in the context of caregivers being less emotionally or physically available to them [38]. This is consistent with caregivers' stress and depressive symptoms being the more important variable explaining emotional and behavioral symptoms as measured by the screening scale in children. Stressed parents might experience greater difficulty in transferring a sense of security and quality bounding to younger children, which has been thoroughly studied in the literature [42]. In adolescents, coping style was the variable with a greater influence on symptoms, with an unproductive style being associated with greater-, whereas a problem-centered style contributing to lowersymptomatology. Other studies have highlighted the relevance of coping strategies as an important resilience related factor for youths $[23,43]$.

The absence of significant differences in socioeconomic status was striking. This was most likely explained by an overrepresentation of high socioeconomic status in our sample, thus with a low power to detect socioeconomic differences. In addition, it is possible that a greater effect might be revealed in the longer term, which will need to be assessed further. Despite the small representation in our sample, the presence of a prior diagnosis of a mental illness predicted an increase in lockdown symptomatology. Preliminary information from clinical samples suggests an impact on this population, particularly in those with an anxiety or attention and hyperactivity diagnosis [27]. Finally, it is also possible that some children and adolescents with social difficulties might have benefited from certain consequences from confinement. Indeed, this might explain the small percentage in our sample (2.1\%) that demonstrated an improvement from baseline to lockdown scores. Positive lockdown consequences have been also reported in adult samples [44].

This study has some limitations to acknowledge. First, there are inherent selection and memory biases in the study, as all the participants were recruited via their own initiative, the information is retrieved partly retrospectively, and represents caregiver reporting. Second, the study has a transversal design and monitorization is required to observe whether these findings will change over time. Finally, despite the fact the sample was enriched with middle-high socioeconomic status Catalan families, we believe it is representative of the same socioeconomic status Spanish population.

\section{Conclusion}


This is one of the first studies to evaluate the risk of psychopathology in children and adolescents during the COVID-19 pandemic lockdown. These data might be used for strategies to detect and prevent those groups at a higher risk of developing a mental illness, and to consider new strategies focused on predictor variables, such as caregiver-focused advertisements [45]. Early interventions in those cases presenting psychopathological symptoms are warranted to prevent later mental health conditions, in this period where stressors are accumulating. Close monitoring at younger ages might be a priority.

\section{Summary}

Multiple stressors are emerging due to the COVID-19 pandemic and related preventive strategies such as lockdown. Substantial efforts are being conducted to balance preventive strategies and potential consequences, for example regarding children and adolescent's mental health. However, this impact has been scarcely studied. We evaluated the risk of psychopathology in children and adolescents during the COVID-19 pandemic lockdown by using a validated scale (PSC), which was administered through an online questionnaire to caregivers of youths between 4-18 years old. We evaluated percentages of at-risk scores during the last year and lockdown, as well as subgroup differences in these lockdown percentages (e.g. age, sex, socioeconomic status, mental health history); a linear regression analysis was used to investigate potential associations between PSC scores and selected variables (stressful life events, coping style in youths, stress and depression in caregivers, youths' history of a mental health illness). We obtained a sample of 1,529 youths, enriched with children representation. In this population, the percentages of at-risk-for-psychopathology scores raised to more than double during lockdown (from $13 \%$ to $34.7 \%$ ), being depression and anxiety the most prevalent symptoms (64.5\%), although hyperactivity and conduct were not negligible (30.8\%). Importantly, children presented a greater risk compared to adolescents $(\mathrm{OR}=1.94)$, particularly those $<8.5$ years old, according to a post-hoc ROC analysis. Stress and depression in caregivers were found to be the best predictors for psychopathological symptoms in children, while in adolescents the best predictor was their coping style (focused on the problem vs. unproductive). These findings suggest that the COVID-19 during the lockdown might have increased the risk for a mental health disorder in youths and particularly in younger ages, despite these findings necessarily need a longitudinal evaluation. Improving caregiver's symptoms and helping adolescents to cope with stress are important targets that might lead to improvement of symptoms. While waiting for studies with larger samples, our results indicate that close monitoring at younger ages and early interventions considering the above-mentioned factors should be a priority in mental health strategies.

\section{Declarations}

Acknowledgements: All the authors of the study thank staff-members of Hospital Sant Joan de Déu, Barcelona who provided valuable feedback. They also thank all the families that voluntarily participated in the study.

\section{Compliance with Ethical Standards}

Conflict of interest. Drs. Via and Dolz are included, respectively, as co-investigator and site IP in a Janssen-Cilag, S.A., clinical trial with the esketamine molecule. All the other authors declare that they have no conflicts of interest.

\section{Ethical Approval}

All procedures performed in studies involving human participants were in accordance with the ethical standards of the institutional and/or national research committee and with the 1964 Helsinki declaration and its later amendments of comparable ethical standards.

\section{Informed Consent}

Informed consent was obtained from all individual participants included in the study

\section{References}

1. World Health Organization (WHO) (2020) WHO I World Health Organization. https://www.who.int/. Accessed 18 Aug 2020

2. Roser M, Ritchie H, Ortiz-Ospina E, Hasell J (2020) Policy Responses to the Coronavirus Pandemic - Statistics and Research - Our World in Data. https://ourworldindata.org/policy-responses-covid. Accessed 18 Aug 2020 
3. Centro Nacional de Epidemiología. Ministerio de Ciencia e Innovación. (2020) COVID-19. https://cnecovid.isciii.es/covid19/. Accessed 13 Aug 2020

4. Holmes EA, O'Connor RC, Perry VH, et al (2020) Multidisciplinary research priorities for the COVID-19 pandemic: a call for action for mental health science. The Lancet Psychiatry 547-560. https://doi.org/10.1016/S2215-0366(20)30168-1

5. Lai J, Ma S, Wang Y, et al (2020) Factors Associated With Mental Health Outcomes Among Health Care Workers Exposed to Coronavirus Disease 2019. JAMA network open 3:e203976. https://doi.org/10.1001/jamanetworkopen.2020.3976

6. Talevi D, Socci V, Carai M, et al (2020) Mental health outcomes of the covid-19 pandemic. Rivista di Psichiatria 55:137-144. https://doi.org/10.1708/3382.33569

7. Wang C, Pan R, Wan X, et al (2020) Immediate psychological responses and associated factors during the initial stage of the 2019 coronavirus disease (COVID-19) epidemic among the general population in China. International Journal of Environmental Research and Public Health 17:. https://doi.org/10.3390/ijerph17051729

8. Zhou J, Yuan X, Qi H, et al (2020) Prevalence of depression and its correlative factors among female adolescents in China during the coronavirus disease 2019 outbreak. Globalization and Health 16:69. https://doi.org/10.1186/s12992-020-00601-3

9. Jones NM, Thompson RR, Schetter CD, Silver RC (2017) Distress and rumor exposure on social media during a campus lockdown. Proceedings of the National Academy of Sciences of the United States of America 114:11663-11668.

https://doi.org/10.1073/pnas.1708518114

10. International Monetary Fund (2020) Spain and the IMF. International Monetary Fund. https://www.imf.org/en/Countries/ESP\#countrydata. Accessed 19 Aug 2020

11. UNESCO (2020) School closures caused by Coronavirus (Covid-19). https://en.unesco.org/covid19/educationresponse. Accessed 13 Aug 2020

12. End Violence Against Children (2020) Home I End Violence Against Children. https://www.end-violence.org/. Accessed 13 Aug 2020

13. Generalitat de Catalunya (2017) Estratègies 2017-2020

14. Danese A, Smith P, Chitsabesan P, Dubicka B (2020) Child and adolescent mental health amidst emergencies and disasters. British Journal of Psychiatry 216:159-162. https://doi.org/10.1192/bjp.2019.244

15. American Psychiatric Association (2013) Diagnostic and Statistical Manual of Mental Disorders (5th ed.). Washington, DC, Arlington, VA: American Psychiatric Association

16. Gunnar MR, Vazquez D (2006) Stress neurobiology and developmental psychopathology. In: Developmental psychopathology: Developmental neuroscience, Vol. 2, 2nd ed. John Wiley \& Sons Inc, Hoboken, NJ, US, pp 533-577

17. Hughes K, Bellis MA, Hardcastle KA, et al (2017) The effect of multiple adverse childhood experiences on health: a systematic review and meta-analysis. The Lancet Public Health 2:e356-e366. https://doi.org/10.1016/S2468-2667(17)30118-4

18. Kessler RC, McLaughlin KA, Green JG, et al (2010) Childhood adversities and adult psychopathology in the WHO world mental health surveys. British Journal of Psychiatry 197:378-385. https://doi.org/10.1192/bjp.bp.110.080499

19. Hawryluck L, Gold WL, Robinson S, et al (2004) SARS control and psychological effects of quarantine, Toronto, Canada. Emerging Infectious Diseases 10:1206-1212. https://doi.org/10.3201/eid1007.030703

20. Sprang G, Silman M (2013) Posttraumatic stress disorder in parents and youth after health-related disasters. Disaster Medicine and Public Health Preparedness 7:105-110. https://doi.org/10.1017/dmp.2013.22

21. Uttervall M, Hultman CM, Ekerwald $\mathrm{H}$, et al (2014) After the flood: Resilience among tsunami-afflicted adolescents. Nordic Journal of Psychiatry 68:38-43. https://doi.org/10.3109/08039488.2013.767373

22. Lenferink LIM, Egberts MR, Kullberg ML, et al (2020) Latent classes of DSM-5 acute stress disorder symptoms in children after single-incident trauma: findings from an international data archive. European Journal of Psychotraumatology 11:1-12. https://doi.org/10.1080/20008198.2020.1717156

23. Duan L, Shao X, Wang Y, et al (2020) An investigation of mental health status of children and adolescents in china during the outbreak of COVID-19. Journal of Affective Disorders 275:112-118. https://doi.org/10.1016/j.jad.2020.06.029

24. Orgiles M, Morales A, Delveccio E, et al (2020) PsyArXiv Preprints | Immediate psychological effects of the COVID-19 quarantine in youth from Italy and Spain. https://psyarxiv.com/5bpfz/. Accessed 6 Aug 2020

25. Mihashi M, Otsubo Y, Yinjuan X, et al (2009) Predictive Factors of Psychological Disorder Development During Recovery Following SARS Outbreak. Health Psychology 28:91-100. https://doi.org/10.1037/a0013674

26. Duan L, Zhu G (2020) Psychological interventions for people affected by the COVID-19 epidemic. The Lancet Psychiatry 7:300-302

Page $11 / 15$ 
27. Jefsen OH, Rohde C, Nørremark B, Østergaard SD (2020) Editorial Perspective: COVID-19 pandemic-related psychopathology in children and adolescents with mental illness. Journal of Child Psychology and Psychiatry jcpp.13292.

https://doi.org/10.1111/jcpp.13292

28. Xiong J, Lipsitz O, Nasri F, et al (2020) Impact of COVID-19 pandemic on mental health in the general population: A systematic review. Journal of Affective Disorders 277:55-64

29. Harris PA, Taylor R, Minor BL, et al (2019) The REDCap consortium: Building an international community of software platform partners. Journal of Biomedical Informatics 95

30. Jellinek MS, Murphy JM, Robinson J, et al (1988) Pediatric Symptom Checklist: Screening school-age children for psychosocial dysfunction. The Journal of Pediatrics 112:201-209. https://doi.org/10.1016/S0022-3476(88)80056-8

31. Hollingshead A. A. (1975) Four-factor index of social status. Unpublished manuscript, Yale University New Haven, C T

32. Lovibond, SH \& Lovibond P (1995) Manual for the Depression Anxiety Stress Scales., 2nd. Ed. S. Sydney

33. Coddington RD (1972) The significance of life events as etiologic factors in the diseases of children-II a study of a normal population. Journal of Psychosomatic Research 16:205-213. https://doi.org/10.1016/0022-3999(72)90045-1

34. Reyes AM, Acuña L (2008) La Escala De Reajuste Social Para Niños. Revista Latinoamericana de Psicología 40:335-344

35. Morales-Rodríguez FM, Trianes MV, Blanca MJ, et al (2012) Escala de Afrontamiento para Niños (EAN): Propiedades psicométricas. Anales de Psicologia 28:475-483

36. World Health Organization (WHO) (2014) Adolescence: a period needing special attention - recognizing-adolescence. https://apps.who.int/adolescent/second-decade/section2/page1/recognizing-adolescence.html. Accessed 20 Aug 2020

37. Murphy JM, Bergmann P, Chiang C, et al (2016) The PSC-17: Subscale scores, reliability, and factor structure in a new national sample. Pediatrics 138:. https://doi.org/10.1542/peds.2016-0038

38. Beesdo K, Knappe S, Pine DS (2009) Anxiety and Anxiety Disorders in Children and Adolescents: Developmental Issues and Implications for DSM-V. Psychiatric Clinics of North America 32:483-524

39. Morrison Gutman L, Joshi H, Parsonage M, et al (2015) Mental health findings from the Millennium Cohort Study Children of the new century Acknowledgements

40. Blair C (2017) Educating executive function. Wiley Interdisciplinary Reviews: Cognitive Science 8

41. Sakyi KS, Surkan PJ, Fombonne E, et al (2015) Childhood friendships and psychological difficulties in young adulthood: An 18-year follow-up study. European Child and Adolescent Psychiatry 24:815-826. https://doi.org/10.1007/s00787-014-0626-8

42. Perry RE, Blair C, Sullivan RM (2017) Neurobiology of infant attachment: attachment despite adversity and parental programming of emotionality. Current Opinion in Psychology 17:1-6

43. Shrivastava A, De Sousa A, Lodha P (2019) Resilience as a Psychopathological Construct for Psychiatric Disorders. In: Advances in Experimental Medicine and Biology. Springer New York LLC, pp 479-489

44. Lau JTF, Yang X, Tsui HY, et al (2006) Positive mental health-related impacts of the SARS epidemic on the general public in Hong Kong and their associations with other negative impacts. Journal of Infection 53:114-124.

https://doi.org/10.1016/j.jinf.2005.10.019

45. Hospital Sant Joan de Déu (2020) Víriques | Faros HSJBCN. https://faros.hsjdbcn.org/ca/tema/viricas. Accessed 23 Aug 2020

\section{Figures}




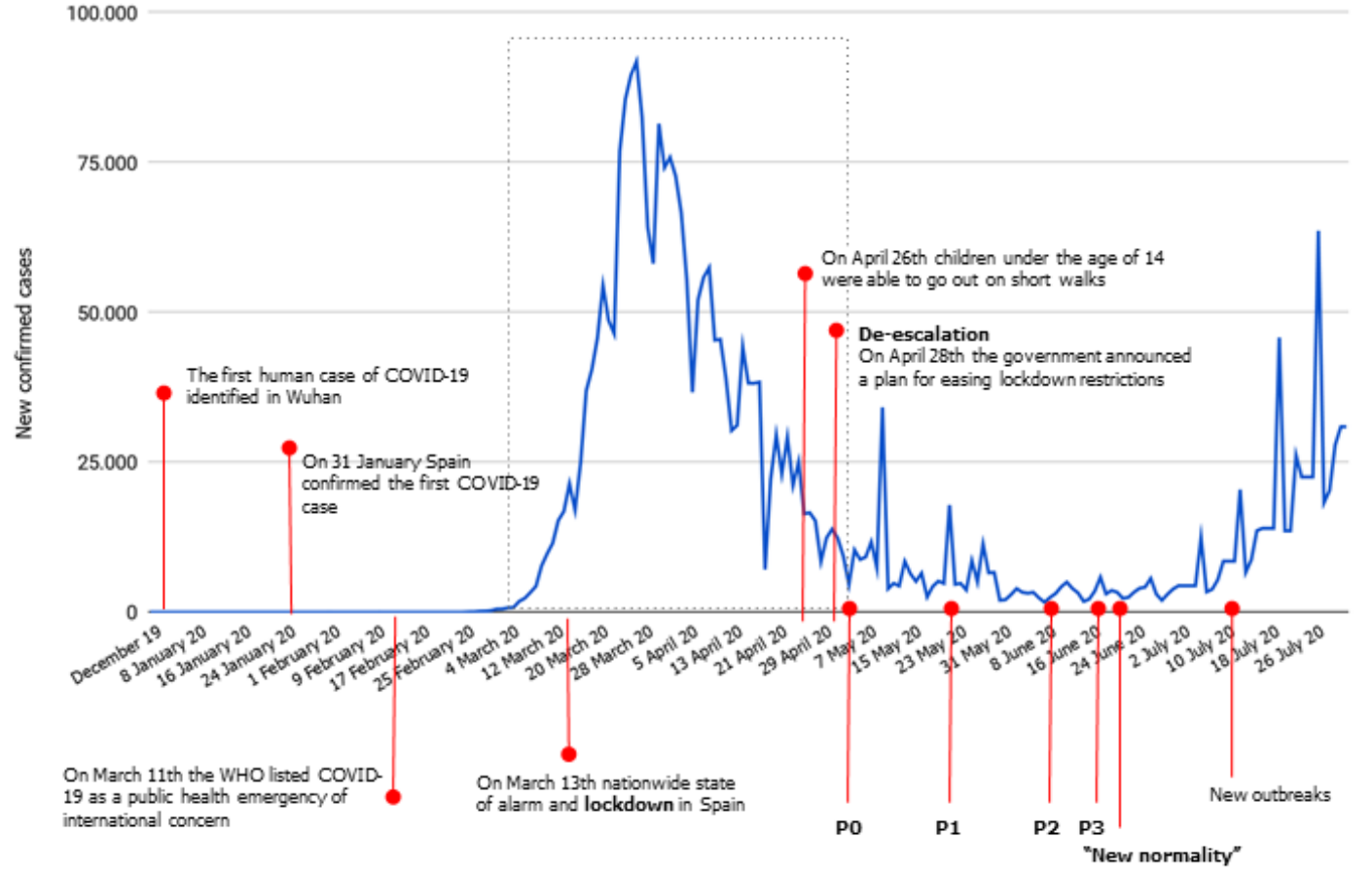

People were allowed to go out for short walks and individual sports on predefined hours of the day.

Schools remain closed.

Phase 1 May 25th - June 8th

Social gatherings allowed up to 10 people. Social distancing measures must be respected at all times between people who do not live together.

Schools remain closed.

Phase 2 June 8th- June 15th

Children and adults allowed to go out for walks and practice individual sports in any part of their province, island or healthcare zone, with no time limit.

Social gatherings allowed up to 15 people. Schools could open only for support activities.

Phase 3 June 15th June 19th

People allowed to move within the province, island or healthcare zone.

"New normality" June 19th

Activities for children and young people could be carried out outdoors but the number of participants were limited to $75 \%$ of their previous capacity.

Schools allowed to open (last day of school term on the 19'l July).

\section{Figure 1}

Timeline diagram of COVID19 new cases and the different phases of lockdown in Spain. A description of the de-escalation is described for Barcelona. Footnote: Source: European Centre for Disease Prevention and Control (ECDC). "New normality": word used by the Spanish Government to define the ulterior to de-escalation phase. There were some regional differences in the velocity of phase changing, we have listed the specifics for Barcelona.

\section{New confirmed cases trend of COVID 19 (Spain)}

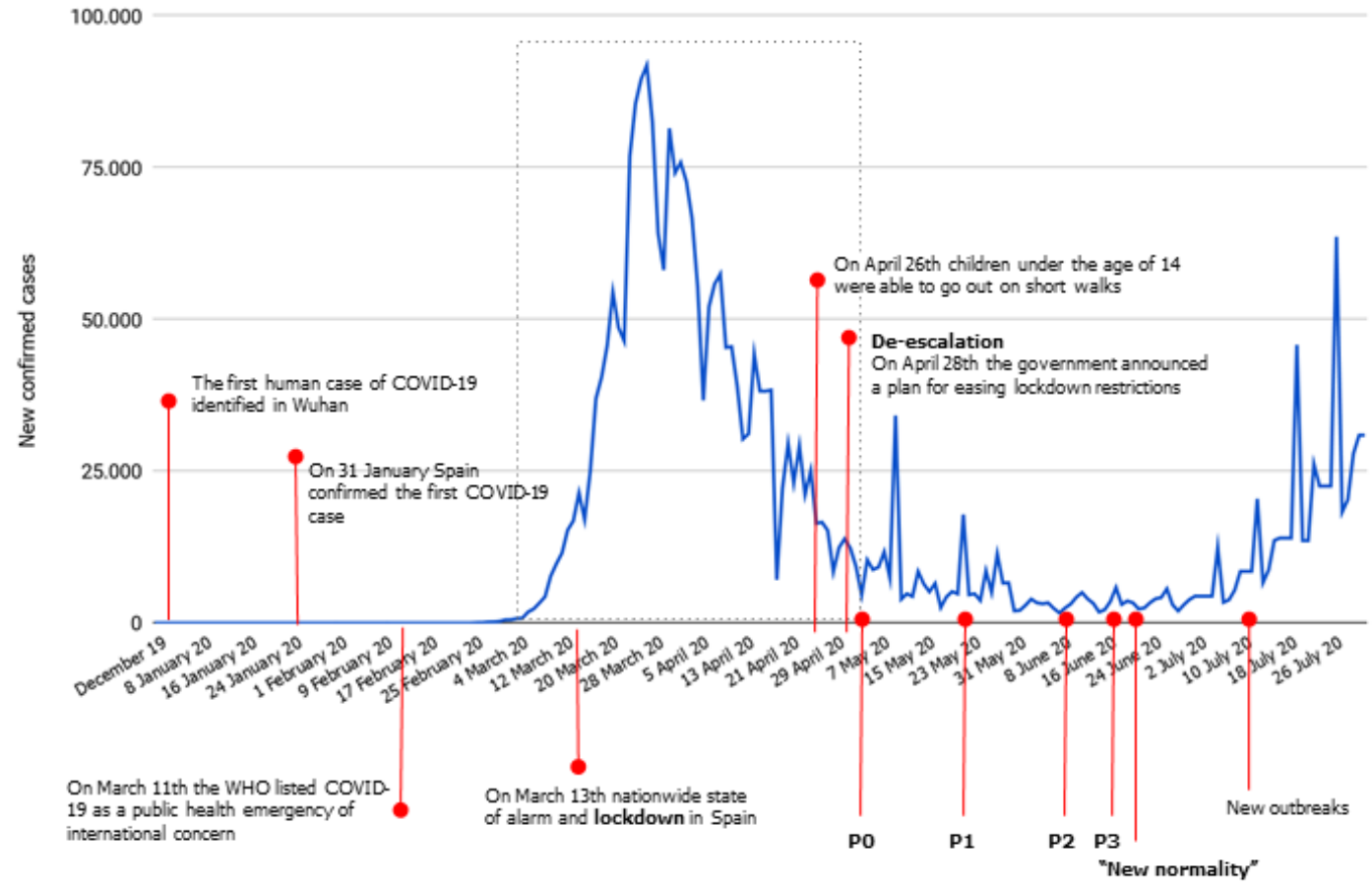

De-escalation (Barcelona, Spain)

Phase 0 May4th- May 18th

People were allowed to go out for short walks and individual sports on predefined hours of the day.

Schools remain closed.

Phase 1 May25th - June 8th

Social gatherings allowed up to 10 people. Social distancing measures must be respected at all times between people who do not live together.

Schools remain closed.

Phase 2 June 8th- June 15th

Children and adults allowed to go out for walks and practice individual sports in any part of their province, island or healthcare zone, with no time limit.

Social gatherings allowed up to 15 people. Schools could open only for support activities.

Phase 3 June 15 th June 19th

People allowed to move within the province, island or healthcare zone.

"New normality" June 19th

Activities for children and young people could be carried out outdoors but the number of participants were limited to $75 \%$ of their previous capacity.

Schools allowed to open (last day of school term on the $19^{\prime \prime}$ July). 


\section{Figure 1}

Timeline diagram of COVID19 new cases and the different phases of lockdown in Spain. A description of the de-escalation is described for Barcelona. Footnote: Source: European Centre for Disease Prevention and Control (ECDC). "New normality": word used by the Spanish Government to define the ulterior to de-escalation phase. There were some regional differences in the velocity of phase changing, we have listed the specifics for Barcelona.

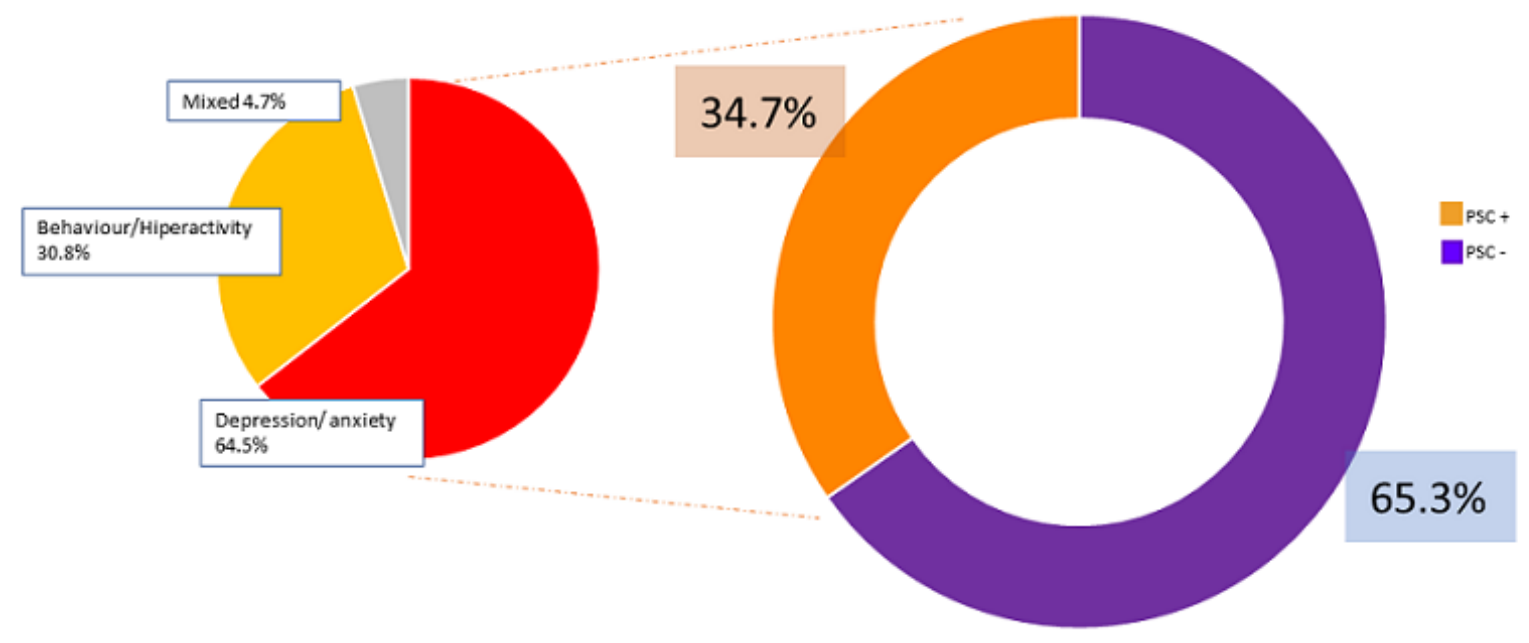

\section{Figure 2}

Circle diagram presentation of PSC+ and PSC- percentages since lockdown. Footnote: PSC+/PSC-: Clinically significant (at-risk)/not significant lockdown PSC (Pediatric Symptom Checklist) scores.

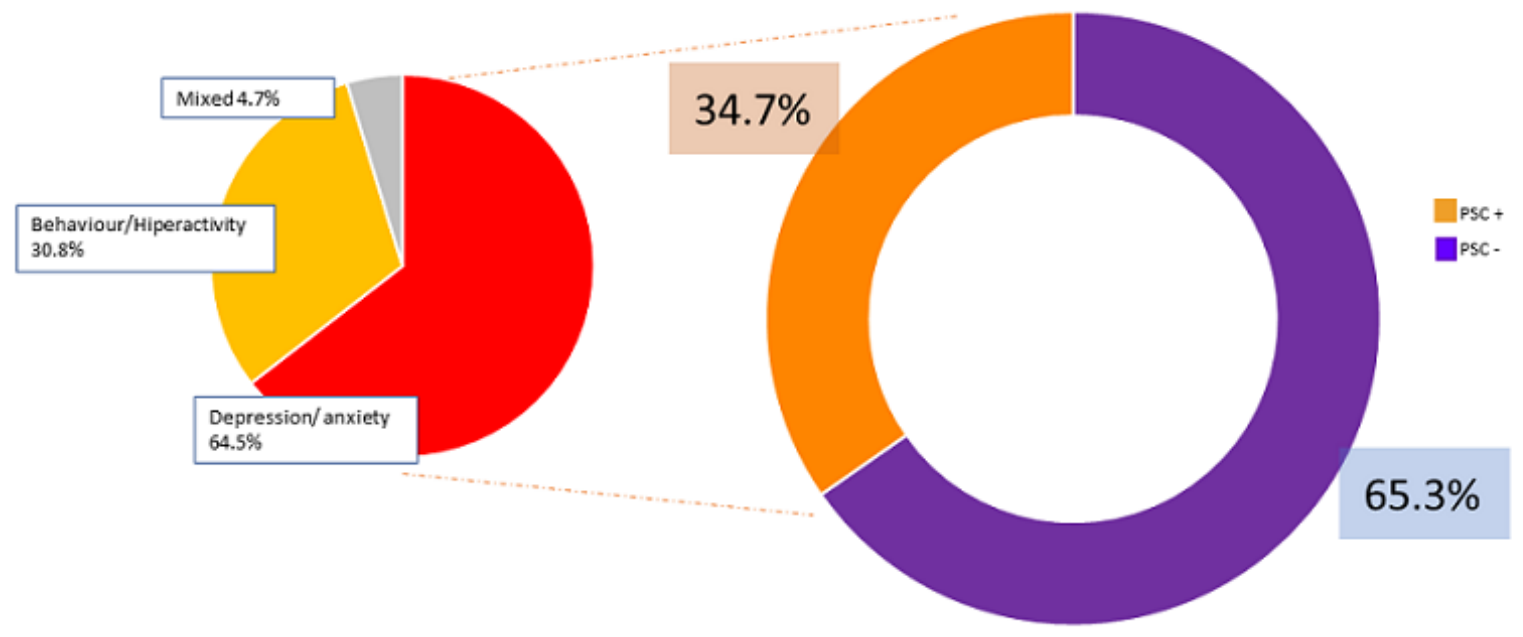

\section{Figure 2}

Circle diagram presentation of PSC+ and PSC- percentages since lockdown. Footnote: PSC+/PSC-: Clinically significant (at-risk)/not significant lockdown PSC (Pediatric Symptom Checklist) scores.

\section{Supplementary Files}

This is a list of supplementary files associated with this preprint. Click to download.

- ViaEstrada2020SupplementaryTablesandFigures.docx

- ViaEstrada2020SupplementaryTablesandFigures.docx

- ViaEstrada2020SupplementaryText1.docx 
- ViaEstrada2020SupplementaryText1.docx

Page 15/15 